\title{
EDITORIAL
}

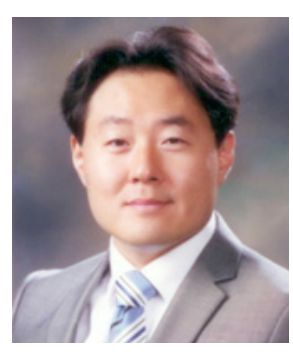

\section{Special Issue on Medical Imaging}

\author{
Dosik Hwang \\ (Co-guest Editor of Special Issue)
}

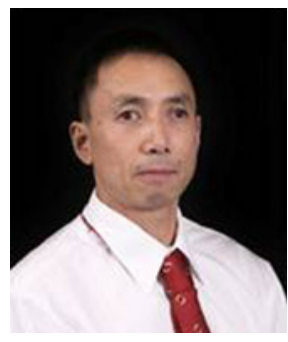

Gengsheng L. Zeng

(Co-guest Editor of Special Issue)

(C) The Korean Society of Medical \& Biological Engineering and Springer 2014

Medical Imaging has been playing one of the most important roles in medical diagnosis. As the technologies advance, so does medical imaging, and it gets an increasing role, not only in diagnosis, but also in treatment, such as image-guided therapies. Medical imaging also has a great impact on many research areas including neuroscience, drug development, phycology, psychiatry, cardiology, and so on. Advancement of the medical imaging technologies involves multidisciplinary research fields that encompass physics, medicine, electrical and mechanical engineering, biology and chemical engineering, etc. Considering the impact of medical imaging on clinics, research, and engineering, we select "Medical Imaging" as the theme of this Special Issue of Biomedical Engineering Letters. This special issue includes five review papers on recent advances of several medical imaging technologies and their applications.

The first review paper entitled, "Model based filtered

Dosik Hwang $(\varangle)$

School of Electrical and Electronic Engineering, Yonsei University, Seoul, Korea

Tel : +82-2-2123-5771

E-mail : dosik.hwang@yonsei.ac.kr

Gengsheng L. Zeng $(\varangle)$

Department of Electrical Engineering, Weber State University, Ogden, Utah 84408 USA

Department of Radiology, University of Utah, Salt Lake City, Utah 84108

USA

Tel : +(801) 626-6864

E-mail : larryzeng@weber.edu backprojection algorithm: a tutorial", by Gengsheng L. Zeng, IEEE Fellow, USA, provides in-depth knowledge of very interesting new type of linear reconstruction algorithm with incorporation of noise models in it [1]. For many decades, the filtered backprojection (FBP) algorithm has been a popular choice in image reconstruction. However, recent developments of new medical imaging systems such as lowdose x-ray CT, nuclear medicine, and real-time MRI applications require more advanced reconstruction algorithms than the conventional FBP in order to incorporate measurement noise and system models. In recent years, many researchers have been developing various iterative algorithms to meet this demand and improve the final reconstructed images. However, the drawback of most iterative algorithms is the heavy computational cost. Therefore, it would be wonderful if we could have an FBP algorithm that performs comparable tasks to the iterative algorithms. In this first review paper, Dr. Zeng provides a way to develop such an FBP algorithm with incorporation of noise models, which may replace the state-of-the-art iterative algorithms.

The second review paper, "Challenges and advances in MR imaging of the intervertebral disc - could the cartilaginous endplate be a biomarker for the disc health?," by Sung M. Moon, Ph.D., GE Healthcare, USA, provides an overview of new magnetic resonance imaging (MRI) techniques and findings on disc disease and cartilaginous endplate (CEP) [2]. A brief description on the anatomy of the CEP and intervertebral disc is followed by their roles in the disc 
degeneration process, and the current diagnostic tools to assess disc health along with the state-of-the-art MR imaging techniques using CEP as a biomarker for accurate diagnosis and prognosis of the disc health.

The third review paper, "A new CT concept: inverse geometry CT," by Jongduk Baek, Ph.D., et. al., Yonsei University, Korea, introduces a new type of CT whose source-detector geometry is inverted compared to the conventional CT by using a small $2 \mathrm{D}$ detector array and multiple x-ray source array, instead of using a single $x$-ray source and a large 2D detector array [3]. Due to its inverted system geometry, it can reduce the cone-beam artifacts caused by insufficient data sampling due to large cone-beam angles in the conventional cone-beam CT. The concept and mechanism of this inverse geometry $\mathrm{CT}$ are explained along with several benefits which include reduced cone-beam artifacts and scatter, isotropic resolution, and high temporal resolution.

The fourth review paper is "Speckle reduction techniques in medical ultrasound imaging," by Yangmo Yoo, Ph.D., et. $a l$., Sogang University, Korea. Ultrasound imaging is one of the most frequently used imaging modalities due to its realtime imaging capability, safety, and easy access. However, its image quality is limited by its low contrast between tissues and speckle patterns due to the interference of backscattered ultrasound waves [4]. This review paper describes three effective methods to reduce speckle patterns, which include frequency compounding, spatial compounding, and post filtering, along with their strengths and weakness.

The last review paper, "Color Doppler twinkling artifact: possible mechanisms and clinical potential," by Min Joo Choi, Ph.D., et. al., Cheju Halla University, Korea, provides an in-depth review on twinkling artifact (TA) which is characterized by a rapidly changing mixture of red and blue colors for a stationary hyper-echogenic target in the color Doppler mode in ultrasound imaging. TA may hamper proper diagnosis of vascular flow, but on the other hand, it may provide useful information for particular pathological targets. This review paper explains possible mechanisms of TA and its clinical potential for diagnosis in various pathological cases [5].

In closing this editorial, we would like to express our deepest gratitude to the authors for providing these highquality review articles on the recent advancement of medical imaging technologies. We also thank many reviewers for their professional comments which strengthened the articles in this special issue. In addition, we would like to express our sincere appreciation to Dr. Jae Sung Lee, Managing Editor, and Dr. Sang-Hoon Lee, Editor-in-Chief of the journal Biomedical Engineering Letters for their help, support, and contribution to this special issue. We hope readers will find this special issue helpful for their future study.

\section{REFERENCES}

[1] Zeng GL. Model based filtered backprojection algorithm: a tutorial. Biomed Eng Lett. 2014; 4(1):3-18.

[2] Moon SM. Challenges and advances in MR imaging of the intervertebral disc - could the cartilaginous endplate be a biomarker for the disc health?. Biomed Eng Lett. 2014; 4(1):1924.

[3] Ryu S, Lee C, Baek J. A new CT concept: inverse geometry CT. Biomed Eng Lett. 2014; 4(1):25-31.

[4] Kang JB, Chang JH, Yoo Y. Speckle reduction techniques in medical ultrasound imaging. Biomed Eng Lett. 2014; 4(1):3240.

[5] Choi MJ, Cho CH, Kang G, Yang J. Color Doppler twinkling artifact: possible mechanisms and clinical potential. Biomed Eng Lett. 2014; 4(1):41-54. 